\title{
La situation linguistique des Juifs et le passage de l'allemand au statut de langue nationale
}

\author{
ULRIKE HASS-ZUMKEHR
}

\section{LANGUE ALLEMANDE ET NATION ALLEMANDE AU XIX ${ }^{e}$ SIÈGLE}

Les langues évoluent toujours en étroite interaction avec l'histoire de la communauté linguistique, c'est-à-dire de la société (voir von Polenz, 1991, 1994 et 1999). C'est pourquoi elles ne se développent pas de façon autonome et à partir de leurs lois immanentes; les éléments décisifs sont plutôt: 1 / la pratique linguistique de groupes sociaux déterminés, 2 / l'influence consciemment exercée par l'État ou certains groupes sur la culture linguistique, et 3 / un cadre idéologique qui attribue à la langue une signification particulière et "supérieure » dans le système de valeurs de la société. On peut encore observer le rôle de ces facteurs à partir des langues des anciens pays colonisés et dans les pays de l'ancien bloc de l'Est.

$\mathrm{Au}$ commencement des langues nationales de l'Europe, on trouve toujours une juxtaposition de variétés régionales d'abord purement orales. Des facteurs culturels et politiques transforment progressivement une juxtaposition horizontale en un spectre vertical de variétés. La domination politique et culturelle d'une région ou d'une couche sociale particulière de même que le passage à l'utilisation écrite de l'une des variétés expliquent que le domaine de validité d'une d'entre elles s'élargit aussi bien sur le plan régional que social et devient dominant, fournissant un modèle stylistique, syntaxique, sémantique, phonétique et orthographique et attribuant aux variétés restantes des niches déterminées en dehors d'elle. Si une variété linguistique se détache de toutes les autres, la juxtaposition horizontale de dialectes et de sociolectes se mue en un spectre organisé verticalement, en une hiérarchie de variétés plus ou moins proches du modèle. Il se constitue une norme linguistique représentée par la variété dominante et permettant de distinguer le juste du faux. Dès qu'un modèle dominant et les normes qui lui correspondent se sont cristallisés, le pouvoir politique 
peut les appuyer et de plus en plus les imposer grâce à des actes souverains, par exemple grâce à des règles linguistiques ou en fondant une Académie.

En outre des représentants de l'élite cultivée peuvent codifier et légitimer la variété dominante dans des grammaires, des dictionnaires et d'autres écrits. Pour que la variété linguistique «élue " conserve son rôle dominant, il faut la soutenir, la cultiver et l'actualiser par des activités que l'on résume en Allemagne sous les concepts de culture linguistique [Sprachkultur und Sprachpflege]. En France l'État s'est occupé de cette tâche grâce à l'Académie française et à l'établissement de règles linguistiques. En Allemagne en revanche ce fut et c'est toujours au mieux le rôle des États fédéraux, mais au fond celui du discours social.

Pour l'allemand, le moment où l'on peut reconnaître la variété dominante suprarégionale intervient quatre cents ou quatre cent cinquante ans après le français, c'est-à-dire vers 1800 . Ici ce ne sont pas les humanistes comme en France mais les représentants des Lumières ou du romantisme qui ont codifié et légitimé la langue. Naturellement les dictionnaires et grammaires élaborés longtemps auparavant ont aussi soutenu la tendance à la formation d'une langue allemande standard, mais les codifications humanistes et baroques étaient en Allemagne restées tributaires des différentes langues régionales et de leur sociolecte. C'est seulement depuis 1800 et pendant tout le $\mathrm{XIX}^{\mathrm{e}}$ siècle qu'on note une action collective pour construire la langue allemande standard dans les Belles Lettres, la science et la technologie. Des interventions de l'État n'ont pu intervenir qu'après la fondation de l'Empire en 1871 ; elles concernaient exclusivement l'orthographe comme les systèmes des mesures, des monnaies et des poids qui existaient en 35 variantes et devaient être unifiés pour des raisons purement économiques.

Ce n'est pas une instance unique qui de la fin du XVII ${ }^{e}$ siècle au $\mathrm{XIX}^{e}$ siècle a imposé la variété dominante en Allemagne mais une conjonction d'instances ou plutôt de modèles : des écrivains d'une région donnée (Meissen, l'Allemagne du Nord), des écrits et des acteurs déterminés (Luther avant tout puis les classiques de Weimar), des institutions dont l'influence s'étendait sur de larges espaces comme les chancelleries et les tribunaux, et les grands dictionnaires ou grammaires. À ces derniers incombait à l'époque des Lumières la tâche de fonder le choix d'un modèle régional et social et d'en démontrer la rationalité. Les auteurs de grammaires et de dictionnaires devaient déduire des usages linguistiques servant de modèles - des usages qui ne pouvaient être unifiés en tous points - les principes du bien écrire et du bien parler permettant d'élargir les possibilités de la langue et de les adapter aux exigences des nouveaux développements sociaux.

Tournons-nous maintenant vers le problème des couches sociales portant la variété dominante. Pour la France, on a établi que jusqu'au $\mathrm{XVI}^{\mathrm{e}}$ siècle l'aristocratie de robe et la riche bourgeoisie fournirent le modèle du «bon usage " mais qu'au XVI ${ }^{e}$ siècle la cour devient la couche sociale 
dominante y compris du point de vue linguistique. C'est elle qui déclencha la fondation de l'Académie française et en précisa les tâches. Le rôle brillant de la cour de France y compris sur le plan linguistique et culturel séduisait aussi les cours des petits États allemands absolutistes du XVII ${ }^{e}$ siècle. Cette " mode » fut de plus en plus critiquée en Allemagne par des représentants de la bourgeoisie. De façon générale la bourgeoisie allemande se développa lentement depuis la fin du XVII ${ }^{e}$ siècle mais devint de plus en plus le vecteur de la culture et donc des préoccupations linguistiques.

Pour la situation linguistique en Allemagne au $\mathrm{XIX}^{e}$ et au $\mathrm{XX}^{\mathrm{e}}$ siècle un autre facteur est extrêmement important : l'idée de l'État nation qui longtemps avant sa fondation, dès la fin du XVIII ${ }^{e}$ siècle, a été formulée par la bourgeoisie et à défaut de réalisation politique a donné lieu à des anticipations dans le domaine culturel. C'est alors que la littérature du classicisme weimarien, la philosophie allemande, l'épanouissement du journalisme, la naissance d'une " opinion publique " jouèrent leur rôle - bref la culture littéraire au sens le plus large dut compenser pendant près de cent ans l'absence d'État-nation bourgeois. La «nation allemande » s'est d'abord constituée sur le plan culturel; la bourgeoisie, en particulier après des revers politiques comme ceux de 1848-1849 a embrassé des idées de nation culturelle dans l'espoir d'atteindre ainsi de l'intérieur l'unité politique de la nation. Un concept dominant dans l'idéologie de la nation culturelle a été forgé par Herder et souvent sollicité, celui d'esprit du peuple. S'y adjoignit le concept employé surtout par les frères Grimm d'esprit de la langue. La langue et l'histoire, ou plutôt la mythologie, n'ont pas seulement constitué l'épine dorsale argumentative dans l'idéologie de la nation culturelle à partir de la fin du XVIII siècle ; la langue et l'histoire étaient aussi au centre de la "religion culturelle " du XIX ${ }^{e}$ siècle et étaient des thèmes favoris de la bourgeoisie cultivée. L'intérêt pour les "vieux germains " et les dialectes qui, étaient considérés comme témoins de la germanité ancienne, originelle, avait des caractéristiques plus ou moins nationalistes. Pour le dire d'un mot, la langue allemande fonctionna durant un temps comme substitut de la nation allemande.

Il fallait naturellement pour cela une image de la langue allemande qui efface tout ce qui résultait de contacts culturels et d'échanges avec d'autres langues et cultures. En particulier l'influence française, associée aux deux traumatismes de la guerre de Trente ans et des guerres napoléoniennes fut niée. Son pendant dans la réflexion linguistique fut le purisme sémantique qui se dirigeait davantage contre les emprunts faits au français que contre ceux faits au latin ou au grec. L'image de la langue allemande était donc orientée de façon téléologique et partiale pour soutenir l'exigence d'une nation allemande.

La culture linguistique chez les représentants d'une idéologie de la culture nationale visait la valeur symbolique de la langue allemande et non les capacités pratiques dont a besoin le membre d'une bourgeoisie économique dans le cadre d'une démocratie parlementaire. L'idée romantique 
d'un esprit de la langue et du peuple excluait que l'on s'occupe de la grammaire ou du style ; l'esprit de la langue, d'après Jacob Grimm ne peut être senti et intuité que par celui qui appartient à la nation allemande. On ne peut le saisir d'un point de vue intellectuel.

Mais la manière romantico-nationale et historique de s'occuper de la langue allemande n'était pas la seule. À côté et dans des îlots en dehors de la philologie allemande continuait à exister un intérêt rationaliste pour la langue, orienté sur les Lumières. Le début du XIX siècle fut non seulement le début de la philologie allemande mais aussi l'époque où s'épanouit la linguistique comparée, le commencement d'un intérêt systématique pour les langues orientales et exotiques. Dans les écoles aussi dominait la traditionnelle relation rationnelle à la langue, qu'il s'agisse de l'enseignement de la langue maternelle ou des langues étrangères; il faut ajouter à cela de plus en plus de réflexions didactiques, la mise en avant des langues étrangères vivantes par rapport aux langues «mortes» du lycée classique.

On ne s'étonne donc pas après ce qui a été dit que les Juifs vivant en Allemagne aient activement participé au développement de la langue nationale allemande, aient mis en relation la haute valeur symbolique de la langue allemande et leur propre situation et se soient sentis liés à l'approche rationaliste du langage, car le mode d'approche nationalromantique les excluait d'emblée de la communauté du peuple.

Ce n'est pas seulement au sein de la germanistique que l'identification du peuple, de l'histoire et de la langue a créé la base de projections réciproques entre ces instances. Une preuve exemplaire en est le discours de l'Académie prononcé en 1851 par Jacob Grimm Sur l'origine du langage où le peuple, l'histoire et la langue coïncident en permanence. Il est une phrase prononcée par Jacob Grimm au congrès des germanistes de Francfort en 1846 que l'on cite souvent en raison de son caractère de sentence définitive :

"Laissez-moi commencer par cette simple question : Qu'est-ce qu'un peuple ? Et répondre de façon tout aussi simple : un peuple est l'essence des hommes qui parlent la même langue. C'est pour nous autres Allemands l'explication la plus naïve et la plus fière" (cité par Grosse, 1985, 481).

Cette phrase et une autre de même contenu' ${ }^{1}$ sont dans une certaine contradiction avec d'autres propos ultérieurs de Grimm qui ont été rassemblés et où Grimm procède, sans même en être sans doute conscient, à une restriction lourde de conséquences de l'équivalence établie entre peuple et communauté linguistique. Il en ressort que Jacob Grimm ne comptait pas les Juifs dans le peuple allemand bien qu'ils fissent partie de la communauté linguistique, bien que même des cercles hostiles à l'émancipation aient cessé vers la même époque de concevoir les Juifs comme

1. «Puisse enfin être reconnue une loi inviolable, selon laquelle tous ceux qui parlent la langue allemande appartiennent aussi au peuple allemand et peuvent dans le besoin compter sur son aide puissante ". (cité par Storost, 1988, 64). 
une nation mais se soient progressivement associés à l'autoperception des Juifs soucieux d'intégration qui ne voulaient - être rien d'autre qu'une "confession" au sein de la nation allemande. L'autoperception des Juifs commença dès les deux premières décennies du $\mathrm{XIX}^{\mathrm{e}}$ siècle à se libérer de la "nation" pour peu à peu se concevoir exclusivement comme une confession. Vers le milieu du siècle, les Allemands étaient pour des Juifs comme Daniel Sanders depuis déjà longtemps "mon peuple ", et l'unité de son peuple était devenue l'une de ses idées-forces.

De facto ce n'était pas du tout le seul lien de la langue qui constituait pour Jacob Grimm l'homogénéité du peuple, comme le pensent beaucoup d'historiens des sciences, par exemple Bahner/Neumann (1985, 28 sq.), quand ils voient le concept de peuple chez Grimm déduit de celui de nation linguistique. Pour Jacob Grimm était considéré en 1848 comme " anti-allemand " quiconque renonçait à la coïncidence de la nation, de l'histoire et de la langue.

Bahner/Neumann (1985) comme Grosse (1985) voient avant tout dans le concept de la nation linguistique une conception antiféodale avec une tradition remontant à Adelung selon laquelle la communauté de langue et d'origine était opposée aux appartenances de castes (Bahner/Neumann 1985, 63 sq.). À l'époque de la naissance de ce topos, c'est-à-dire chez Herder, Adelung et d'autres représentants des Lumières, les Juifs ne pouvaient pas encore se sentir intégrés du point de vue de la nation linguistique. Que les Juifs soient une nation correspondait à l'usage linguistique des Lumières et, vu la séparation qui prévalait alors entre le monde des Allemands et des Juifs, à une certaine réalité. Mais à l'époque de Grimm les Juifs n'étaient pas une nation qu'on pût distinguer du point de vue de la communauté linguistique de la nation allemande.

Dans la génération des Grimm et celle de leurs successeurs étaient formulées des hypothèses qui refusaient ou du moins limitaient fortement aux minorités polyglottes l'appartenance au peuple allemand. La même observation vaut pour des groupes à l'histoire partiellement divergente. De tous temps des migrations sociales ont franchi dans les deux sens les frontières de l'espace linguistique et de l'espace d'établissement des Allemands entraînant, surtout sur les marges géographiques, des situations de plurilinguisme. Les Juifs n'étaient pas les seuls.

PERSPECTIVES EXTERNES ET INTERNES

SUR LA RELATION DES JUIFS À LA LANGUE ALLEMANDE

\section{L'acculturation par la langue}

La relation particulière qui vient d'être esquissée entre le nationalisme allemand et la fonction symbolique de la langue allemande a conduit les représentants de l'émancipation juive comme Dohm et les auteurs des 
édits d'émancipation à l'association d'idées suivante : l'acculturation est la condition préalable pour assurer l'émancipation - une culture allemande est le présupposé de l'acculturation - la culture, c'est-à-dire la compétence linguistique est le présupposé de base pour tout autre degré de la culture.

Au plus tard depuis les années 1830 l'allemand n'était plus pour la majorité des Juifs vivant en Allemagne une langue étrangère ou une seconde langue mais avait relayé le yiddish comme langue maternelle (Kiefer, 1985, 1202). Encore à l'époque des Lumières, durant la seconde moitié du XVIII e siècle, seuls pouvaient parler et écrire allemand les Juifs qui entraient en contact immédiat avec l'environnement germanophone, par exemple les Juifs de cour ou les grands marchands. L'exigence que les rabbins connaissent l'allemand et que les livres commerciaux soient tenus en allemand, une exigence qu'on rencontre sans cesse dans les "règlements concernant les Juifs » du XVIII ${ }^{e}$ siècle, le prouve bien ${ }^{\prime}$. Depuis que Moses Mendelssohn, le symbole d'une émancipation aussi linguistique par rapport à la langue maternelle yiddish, devint l'un des meilleurs stylistes allemands de son temps, la relation des Juifs à la langue allemande a commencé à se modifier rapidement. En quelques décennies, l'allemand atteignit le statut de langue maternelle d'abord dans les villes, puis aussi à la campagne et dans les couches sociales inférieures, un processus qui était achevé au plus tard dans les années 1830 et 1840, même si l'on doit ici partir d'un profond fossé entre pauvres et riches, et entre villes et campagnes, et d'un décalage correspondant dans le processus. Dans les biographies de Heinrich Heine (Krohn, 1967, 29 sq.), de Moses Mendelssohn, Rahel Varnhagen et Daniel Sanders, on trouve des exemples d'un passage en deux à trois générations du yiddish occidental à la langue maternelle allemande.

Pour une élite cultivée plus réduite qui n'a pu d'abord se constituer que dans les familles les plus fortunées, l'allemand allait de soi dès le tournant du siècle. L'allemand était davantage encore un symbole hautement valorisé de l'intégration culturelle et nationale à laquelle les Juifs aspiraient. Le processus de dénationalisation et de confessionnalisation des Juifs allemands est très étroitement lié à l'appropriation de la variété linguistique dominante en Allemagne et à une maîtrise supérieure à la moyenne. Les efforts de formation de la communauté juive et de nombreux individus, notamment dans les familles des Juifs de cour et dans les villes ont éventuellement abouti à ce qu'au moins quelques Juifs, de Mendelssohn à Rahel Varnhagen ont maîtrisé la variété dominante de la langue nationale allemande plus tôt et mieux que la plus grande partie des Allemands non juifs. De façon générale, les Juifs se sont manifestement plus orientés que les non-Juifs sur les variétés linguistiques suprarégionales et davantage valorisées (Jeggle, 1969, 237).

1. Par exemple dans Löwenstein, 1895, 226; Gebhardt, 1903, 97-113 ; Stern, 1971, 1 : Hlbbd., 111 sq., 563, 578 ; 2 : Hlbbd., 987 sq., 1030, 1297, 1576, 1581 ; Karniel, 1982, 73 sq. 
Les édits d'émancipation à partir de la fin du XVIII ${ }^{\mathrm{e}}$ siècle prévoyaient en règle générale que les Juifs devaient tenir leurs livres de commerce et aussi les livres de gages en langue et en écriture allemande, et que des noms de famille allemands devaient être adoptés (Bering, 1988) - cela aussi était lié à un changement du système d'écriture bien que dans la communication interne à la communauté juive l'allemand ait été aussi écrit en caractères hébraïques (Freimark, 1979). En outre la formation des rabbins fut associée à l'étude dans une faculté de philosophie. Bien des Juifs crurent eux-mêmes à la voie royale de "l'égalité par la culture linguistique ». Très importantes furent les fondations d'écoles juives suscitées par les Lumières qui eurent lieu depuis la fin du XVIII et dans la première moitié du XIX ${ }^{e}$ siècle, des écoles où pour la première fois formation séculière et langue allemande se trouvaient au premier plan, même si les contenus éducatifs traditionnellement juifs - la bible hébraïque dans le texte original et le Talmud, c'est-à-dire une littérature de commentaire furent plus ou moins conservés en marge'.

Même dans la réforme du service divin des synagogues le remplacement de parties hébraïques de la liturgie par des parties allemandes joua un rôle important. Des controverses eurent lieu pour savoir si la prédication et les prières devaient être prononcées en langue allemande (Dubnow, 1929, 42 sq., 86 sq., 165 ; Wiener, 1933, 99 sq.).

À la fin du XIX ${ }^{\mathrm{e}}$ siècle l'acculturation et l'assimilation ont abouti ici et là à une teutomanie linguistique, par exemple au consistoire central qui s'aligna sur le purisme de la société wilhelminienne (Paucker, 1976, 526).

\section{Le yiddish}

Le bon allemand est opposé à la langue que les Juifs devraient abandonner en même temps que leur existence dans le ghetto, langue que l'on appelait à l'époque leur "jargon » leur Mauscheln ou l' «allemand des Juifs ", le "judéo-allemand ». Cette langue ne s'appelle le yiddish chez les linguistes que depuis le $\mathrm{Xx}^{\mathrm{e}}$ siècle. Les termes de judéo-allemand, d'allemand hébraique, d'allemand des Juifs servaient à désigner le groupe de dialectes du yiddish occidental dans l'espace ouest-européen et allemand de l'époque (Kiefer, 1985, 1202 ; voir von Polenz, 1991, 293-298). L'évaluation de cette langue était à peu près identique chez les non-Juifs et chez les Juifs en mal d'émancipation : le judéo-allemand serait une barrière à l'émancipation. Par judéo-allemand on pouvait au XIX siècle entendre des réalités différentes : a) l'allemand écrit en caractères hébraïques; b) l'allemand parlé avec des interférences plus ou moins fortes de yiddish;c) l'ar-

1. On trouve des informations fondamentales chez Freund, 1908 ; Rosenthal, 1934 ; Kurzweil, 1987 ; Aschkewitz, 1967. Richartz fournit un aperçu sur le passage de la forme scolaire du Cheder traditionnel au XVIII ${ }^{c}$ siècle à l'école moderne à partir du début du XIX ${ }^{c}$ siècle (1975, 51 sq.). Essentiel pour le thème de l'école juive dans la période de transition Eliav (1960). 
got à l'emploi duquel les non-Juifs associaient de fortes intentions conspiratives ; et d) une manière de parler, d'écrire et de penser caractérisée par le monde environnant comme «calculée » et «talmudique » (voir Freimark, 1979).

Aujourd'hui encore le yiddish est souvent présenté comme un mélange mis en relation trop étroite avec l'allemand, à côté duquel il apparaît alors comme en décalage et déficitaire (Kiefer, 1985, 1202). Le fort intérêt du $\mathrm{XIX}^{\mathrm{e}}$ siècle pour les dialectes allemands fortement valorisés ne s'est nullement appliqué au yiddish qui même chez les Juifs assimilés a été largement dévalorisél.

Tout particulièrement l'argot mis en étroite relation avec le yiddish fut considéré comme une imitation dérisoire de la langue allemande et - par une fréquente projection - comme une menace pesant sur la "germanité ». Ici s'opérait une identification sociale au moins partielle des Juifs avec des criminels. Jeggle (1969) a constaté que dans l'environnement allemand des séries d'expressions relevant de l'argot yiddish étaient universellement répandues et ne pouvaient donc en aucun cas constituer un moyen de communication secret.

Pour Jacob Grimm aussi le judéo-allemand «défigurait » à la fois l'allemand et l'hébreu. La dévalorisation du yiddish n'avait pas que des causes sociales et n'était pas seulement un reflet des conditions sociales dans les conditions linguistiques. L'importance que la linguistique historicocomparative accordait à l' "indo-européen " pour la refuser à toutes les autres langues et donc aussi à la famille des langues sémitiques, devrait avoir contribué à l'image du yiddish (voir von Polenz, 1991, 293 sq. ; Bering, 1991, 329).

\section{Pas d'accès à l'esprit de la langue}

La maîtrise supérieure à la moyenne de la variété linguistique dominante et suprarégionale de l'allemand à laquelle étaient parvenus les Juifs n'apporta pas l'intégration escomptée, au contraire. La société en voulut aux Juifs d'avoir "fait ce qu'il fallait au-delà des normes" et les philologues livrèrent les arguments appropriés. Après l'identification propagée par Jacob Grimm du peuple et de la communauté linguistique (voir supra), les Juifs auraient dû, au plus tard vers 1830, être considérés comme appartenant au peuple allemand. Mais les germanistes du XIX ${ }^{e}$ siècle ne tirèrent pas cette conclusion et en restèrent à leur concept ethnique du peuple.

Un facteur important, à l'arrière-plan de l'opinion publique et des germanistes universitaires, est constitué par les représentations concernant les capacités linguistiques des Juifs et leur influence négative sur la langue allemande. La haute compétence linguistique manifestement atteinte fut dis-

1. L'article "Judéo-allemand" de I. M. Jost dans Ersch/Gruber, Encyclopédie universelle des sciences et des arts, section 2, $27^{\mathrm{c}}$ partie, Leipzig, 1850, p. 322-324 est un bon exemple de cette position. 
créditée avec l'aide du concept d'esprit de la langue comme étant une imitation superficielle derrière laquelle se cachait le "parler juif», impossible à perdre et tout au plus dissimulé. Si les penseurs des Lumières avaient exigé que la langue allemande soit maîtrisée, les romantiques stigmatisèrent précisément cette compétence en la considérant comme "dépourvue d'esprit " et en y voyant un manque du sens de la langue et de la nation.

Intéressante est dans ce contexte aussi l'attitude des professeurs prussiens qui s'exprima en 1846 dans un questionnaire portant sur l'autorisation éventuelle d'enseigner accordée aux Juifs dans les différentes philologies. L'arrière-plan était le pourcentage disproportionné d'étudiants juifs au milieu du $\mathrm{XIX}^{\mathrm{e}}$ siècle ${ }^{1}$. En 1847 la diète prussienne conféra de nouveau sur la "question de l'émancipation des Juifs" et de l'ouverture des branches professionnelles qui leur étaient fermées depuis 1823. Mais le décret projeté comportait d'emblée d'importantes restrictions : là où les statuts d'une université prescrivaient que ses membres pour les disciplines comme les mathématiques, les sciences de la nature et la médecine devaient appartenir à la confession chrétienne, ces statuts conservaient leur validité. Pour les facultés de droit et de théologie, on partait de toute façon d'une évidente incompatibilité en raison d'un manque d'affinité intellectuelle. On argumentait de la même manière dans la faculté de philosophie pour la philologie classique, l'histoire et la philosophie ; les objets de ces sciences ne pouvaient être finalement compris que dans la perspective d'une vision du monde chrétien. Malgré ces restrictions drastiques à l'accès des Juifs aux universités, celles-ci protestèrent si bien que le ministre prussien Eichhorn durant l'hiver 1847-1848 demanda aux professeurs ordinaires de toutes les universités prussiennes de prendre position. Il s'agissait de savoir si les Juifs, d'après les mesures modérées prises dans le nouveau texte de loi, pouvaient être autorisés à enseigner en tant que privatdocents, assistants ou professeurs en médecine, dans les sciences de la nature, en géographie et en philologie. Sur les 280 réponses envoyées $40 \%$ des réponses individuelles et $50 \%$ des réponses de facultés étaient opposées à l'ouverture (Schorsch, 1980, 11). Dans notre contexte sont particulièrement intéressantes les réserves exprimées par de nombreux professeurs à l'idée d'ouvrir aux Juifs les sciences du langage en raison de la relation entre philologie, exégèse et "disposition mentale ».

Les arguments réitérés dans les réponses se meuvent pleinement dans le cadre de stéréotypes sociaux: indépendamment d'une "nature étrangère " sans cesse constatée on fondait le refus des enseignants juifs en alléguant leur égoïsme, leur dégénérescence morale, leur hostilité fanatique aux chrétiens (chez les Juifs traditionalistes), leur athéisme (chez les Juifs libéraux), leur mainmise sur la presse, leur caractère importun et sans scrupules. La tournure que le sujet avait prise dans les têtes était celle d'un

1. Rapporté dans la suite de l'article d'après Schorsch, 1980, avec de nombreuses indications de sources et de bibliographie; voir Richarz, 1982. 
judaïsme "pénétrant » l'université, non pas celle de l'intégration de personnalités scientifiques de confession ou d'origine juive (ibid., p. 12 sq.).

En 1852 le ministre prussien Karl Otto von Raumer argumenta de nouveau en faveur du caractère chrétien de l'Université prussienne et refusa la candidature du philologue classique Jacob Bernays, malgré la grande qualité des attestations en disant que précisément la formation classique de futurs professeurs de lycée et de théologiens " ne doit pas être en opposition à la mentalité et au mode de pensée des chrétiens, mais doit être un moment essentiel de son renforcement ». Or un enseignant de confession juive serait "hors d'état d'offrir les garanties nécessaires pour former des professeurs chrétiens dans des lycées chrétiens" (cité par Schorsch, 1980, 19).

\section{Polylinguisme}

Pendant la période de transition où la langue maternelle est passée du yiddish à l'allemand s'est nécessairement instaurée dans la communauté juive une forte compétence au polylinguisme. Provisoirement on peut seulement formuler comme hypothèse qu'à côté du yiddish, de la variété dominante de l'allemand, du dialecte régional allemand et de l'hébreu les Juifs cultivés semblent avoir très tôt dominé principalement les langues vivantes. Le philologue et philosophe de la langue Heymann Steinthal se souvenait de sa jeunesse au début des années 1830 en ces termes:

"Nous autres, les enfants, nous connaissions une quadruple forme de l'allemand: Nos parents parlaient le judéo-allemand proprement dit, parsemé de mots en hébreu, qui dans la langue courante étaient souvent prononcés autrement que dans la prière en hébreu. Les garçons chrétiens parlaient un dialecte d'Allemagne centrale. Nous, les enfants juifs, nous ne parlions ni comme nos pères et mères, ni comme les enfants chrétiens, qui naturellement s'exprimaient exactement comme leurs parents chrétiens. C'était un judéo-allermand modéré. Je dois ajouter, que beaucoup de Juifs et Juives âgés possédant une certaine culture, par exemple mon père qui avait même appris le français et écrivait un allemand assez correct, parlaient dans leurs relations avec les chrétiens exactement comme ces derniers : dialecte avec les simples citoyens; un allemand pur ou tendant à la pureté avec les notables " (Steinthal cité dans Gay, 1976, 256).

On sait qu'Henriette Herz ou Rahel Varnhagen avaient des compétences comparables dans les langues étrangères (Thomann Tewarson, 1987). Celui qui domine plus de trois langues s'intéresse par la suite sans doute plus facilement aux questions de linguistique comparée.

\section{Activités traductrices}

C'est en liaison avec l'extension du plurilinguisme parmi les Juifs qu'il faut voir leur activité de traducteurs et d'interprètes, c'est-à-dire au $\mathrm{XVIII}^{\mathrm{e}}$ siècle surtout chez les rabbins. Leur rôle d'intermédiaires entre les communautés juives et le monde environnant germano-chrétien fut 
d'abord et essentiellement une médiation entre mondes linguistiques; à l'allemand, à l'hébreu, au yiddish occidental et oriental s'ajoute encore dans les affaires juridiques du XVIII ${ }^{\mathrm{e}}$ siècle le latin. On faisait appel aux compétences linguistiques des rabbins surtout devant les tribunaux et sur les marchés où venus du centre et de l'est de l'Europe les non-Juifs et les Juifs se rassemblaient (Stern, 1971, 2e section, I ler demi-tome, 548, 604 sq., 1581) avec des connaissances linguistiques partiellement incompatibles. Des Juifs baptisés travaillaient dans la seconde moitié du XVIII ${ }^{e}$ siècle comme traducteurs pour le gouvernement prussien (Blank, 1940). Blank (1940) évoque des affaires juridiques traitées entre des marchands juifs en Pologne et en Lithuanie; les Juifs lithuaniens et polonais pouvaient se comprendre oralement en allemand, mais par écrit seulement en yiddish et en hébreu (voir Stern, 1971, $1^{\text {re }}$ section).

L'activité de Moses Mendelssohn comme traducteur servit plus d'une fois à repousser les reproches selon lesquels les prières juives contenaient des déclarations hostiles aux chrétiens. Naturellement il dut dans ce contexte se confronter avec les traductions de Luther, surtout celles des psaumes (Stern, 1971, $1^{\text {re }}$ section, 338 sq.) et réfléchir sur le processus de la traduction. La pratique encore largement répandue au XVIII ${ }^{\mathrm{e}}$ siècle de la lecture publique de nouvelles lois et de nouveaux traités conduisit à ce que les Juifs demandèrent une communication écrite, car il la fallait pour qu'ils puissent exécuter des traductions et en rendre le contenu accessible à leur propre communauté (Stern, 1971, $1^{\text {re }}$ section). Le plurilinguisme et l'activité traductrice allaient ainsi de pair avec une tendance à la transcription d'une communication d'habitude orale et ont exercé de la sorte une pression sur l'alphabétisation de la population juive moyenne qui, comme les couches correspondantes non juives, n'aurait pas été alphabétisée jusqu'à une période avancée du XIX ${ }^{\mathrm{e}}$ siècle.

$\mathrm{Au} \mathrm{XIX}^{\mathrm{e}}$ siècle beaucoup de Juifs cultivés ont tenté d'exploiter leur acculturation en traduisant de la littérature juive en allemand, sans doute dans l'espoir de convaincre la société allemande de la valeur de leur propre culture.

\section{Philologues et linguistes}

Les professeurs des écoles juives étaient aussi durant le $\mathrm{XIX}^{\mathrm{e}}$ siècle comme leurs collègues des écoles non juives actifs comme chercheurs et publiaient par exemple dans des programmes scolaires. Le commun dénominateur de leur intérêt pour les langues et la didactique des langues était une théorie linguistique rationaliste et antiromantique. Les questions analytiques, les thèmes comparatistes et le succès didactique en sont les traits dominants'. De façon explicite Lazarus Geiger (1870) et Daniel Sanders

1. Voir le titre de I. M. Jost, Lehrbuch des hochdeutschen Gedankenausdrucks in Wort und Schrift und die Schule des freien Gedankenausdrucks (paru après 1834; voir Hess, 1857, 36). 
(voir Hass-Zumkehr 1995) se sont tournés contre la théorie linguistique dominante de la philologie allemande représentée par Jacob Grimm et son intérêt pour l'étymologie et l'histoire de la langue. Même la mise en évidence de processus d'échange entre les langues (par exemple entre l'hébreu et le grec chez Sanders) s'opposait à la théorie linguistique de l'histoire nationale.

\section{Conceptions de la langue}

La très nette prédilection des Juifs pour une conception de la langue poursuivant les traditions des Lumières et le refus en partie véhément, voire la lutte contre l'étude de la langue de type national et romantique, seront en conclusion examinés du point de vue de leurs fondements à partir de l'exemple de Berthold Auerbach.

Auerbach a consacré un espace démesuré à la "formation du discours " dans son livre paru en 1843 sur le " citoyen cultivé ». " La formation du discours » est subsumée sous le thème de la "formation de la connaissance » et distinguée de la "formation par les livres». Auerbach met en évidence les motifs pour lesquels la formation linguistique et la culture sont identifiées l'une à l'autre : le progrès de la société civile en termes de civilisation et en même temps de politique en était réduit à la capacité de l'individu à communiquer. C'est lié chez lui à un mélange de réalisme linguistique et d'idéalisme : les idées et les perspectives sont là et doivent être exprimées (réalisme), mais le fait d'exprimer éclaire et développe de façon essentielle l'aptitude à penser et à sentir (idéalisme). De la sorte le développement des capacités linguistiques réalise un progrès dans la formation de l'individu (et de la nation ou de l'humanité comme totalité des individus). Si l'on ajoute les observations d'Auerbach sur l'amour de la patrie - qui jouent également un rôle important dans le concept cadre de "citoyen cultivé », deux conceptions de la formation linguistique se manifestent: la priorité revient à l' " autodétermination " de l'individu en relation à " son discours ", c'est-àdire au choix des expressions adaptées et "claires ", c'est-à-dire assurant la communication. Ce n'est que si l'autodétermination réussit au niveau de l'individu-citoyen qu'elle réussit aussi dans la vie sociale et politique. La langue est ici principalement comprise comme un instrument, même si à côté de cela on fait allusion à des idées romantiques courantes comme l'amour de la langue maternelle allemande. La conception dominante de la formation linguistique s'exprime chez Auerbach comme suit :

" De même que l'homme doit en tous points guider et dominer sa nature, il doit le faire en particulier dans la langue. On ne doit pas toujours se laisser simplement aller, mais il faut se guider et s'orienter soi-même [...] en changeant l'expression, en choisissant telle ou telle d'entre elles, on exerce une autodétermination sur son discours qui doit être développée et formée [...] Le but du langage est d'être compréhensible à d'autres. Nous, les Allemands, sommes la nation qui a la plus profonde pensée, mais en termes de dextérité et d'habileté du discours nous sommes derrière des autres nations cultivées ; $[\ldots]$ » 
Dans la formation du discours se manifeste le plus clairement la force de l'observation de soi-même, de l'autodétermination et de l'autoformation [...] Chaque citoyen a besoin d'une certaine habileté à parler et chacun peut l'acquérir s'il veille simplement à sa manière habituelle de parler et veille précisément à dire totalement et réellement ce qu'il voulait dire [...] Chacun peut ainsi conquérir la situation qui correspond à sa formation. Mais même sous d'autres rapports [...] la formation du discours est d'une immense importance [...] dès maintenant nous sommes souvent conduits dans nos cercles professionnels, dans nos associations de bienfaisance, de soutien des arts, dans nos chorales, à nous exprimer publiquement. [...] plus le bon sens naturel et le libre talent oratoire se développeront dans une bourgeoisie libre et indépendante, plus la reconnaissance et la participation des citoyens dans toutes les affaires publiques seront durables (Auerbach, 1843, 39-45)

C'est ici que les objectifs de la formation linguistique auxquels la majorité des Juifs éclairés du $\mathrm{XIX}^{\mathrm{e}}$ et $\mathrm{du} \mathrm{XX}^{e}$ siècle se savaient redevables s'expriment clairement : la participation à la discussion publique de sujets politiques, sociaux, culturels et économiques. La conception juive de la formation linguistique accompagna l'émancipation politique de la bourgeoisie à laquelle les Juifs espéraient être associés.

Institut für deutsche Sprache D. 68016 Mannhein

\section{LITTÉRATURE}

Aschkewitz, Max, Zur Geschichte der Juden in Westpreußen, Marburg, 1967.

Auerbach, Berthold, Der Gebildete Bürger, Carlsruhe, 1843.

Bahner, Werner / Neumann, Werner (Hgg.), Sprachwissenschaftliche Germanistik, Ihre Herausbildung und Begründung, Berlin, 1985.

Bering, Dietz, Der Name als Stigma. Antisemitismus im deutschen Alltag, 1812-1933, Stuttgart, 1988.

Blank, Heinrich, «Die Übersetzer für Hebräisch und Jiddisch in Königsberg in der zweiten Hälfte des 18. Jahrhunderts ", in Mitt. des Vereins für die Geschichte von Ost- und Westpreußen, Jg., 15, 1940, 1-7.

Dubnow, Simon, Die neueste Geschichte des jüdischen Volkes. Das Zeitalter der ersten Emanzipation (17891815); übers, von A. Steinberg, Berlin, 1928.

Eliav, Mordechai, "Jüdische Erziehung in Deutschland im Zeitalter der Aufklärung und der Emanzipation ", in Bulletin des Leo-Baeck-Instiututs, Nr. 11/1960, 207-215.

Freimark, Peter, "Language Behaviour and Assimilation. The Situation of the Jews in Northern Germany in the First Half of the Nineteenth Century ", in Leo-Baeck-Institute-Year Book, 24/1979, 157-177.

Freund, Ismar, Die Rechtsstellung der Juden im preußischen Volksschulrecht, Berlin, 1908.

Gay, Peter, "Begegnung mit der Moderne. Deutsche Juden in der deutschen Kultur ", in E. Werner Mosse, Arnold Paucker (Hgg.), Juden im Wilhelminischen Deutschland, 1890-1914, Tübingen, 1976, 241-311.

Gebhardt, Bruno (Hg.), Wilhelm von Humboldts Politische Denkschriften, 1. Bd., 1802-1810, Berlin, 1903.

Geiger, Lazarus, "Ueber deutsche Grammatik als Lehrgegenstand an deutschen Schulen ", in Einladungsschrift zu der am 4., 5., 6 und 7. April 1870 stattfindenden öffentlichen Prüfung der Real = und Volks $=$ Schule der israelitischen Gemeinde, Frankfurt am Main, 1870, 3-32. 
Grosse, Rudolf, "Volk und Nation bei Grimm und seinen Nachfolgern "; in Zeitschrift für Phonetik, Sprachwissenschaft und Kommunikationsforschung, 38/1985, 481-488.

Hass-Zumkehr, Ulrike, Daniel Sanders. Aufgeklärte Germanistik im 19. Jahrhundert, Berlin, 1995.

$\mathrm{Heß}$, Michael, Die Bürger- und Realschule der israelitischen Gemeinde zu Frankfurt am Main von ihrer Entstehung im Jahre, 1804 bis zu meinem Abtreten von derselben im Juli, 1855, Francfort-sur-le-Main, 1857.

Jeggle, Utz (1969), Judendörfer in Württemberg, Tübingen, 1969.

Karniel, Josef, "Das Tolerenz-Patent Kaiser Josephs II für die Juden Galiziens und Lodomeriens ", in Jahrbuch des Instituts für Deutsche Geschichte XI, 1982, Tel Aviv, 55-89.

Kiefer, Ulrike "Das Jiddische in Beziehung zum Mittelhochdeutschen", in Sprachgeschichte. Ein Handbuch zur Geschichte der deutschen Sprache und ihrer Enforschung, 2 Halbbände, hg. von Werner Besch, Oskar Reichmann, Stefan Sonderegger, Berlin, 1984/1985, 2. Halbbd., 1201-1210.

Kurzweil, Zwi Erich, Hauptströmungen jüdischer Pädagogik in Deutschland von der Aufklärung bis zum Nationalsozialismus, Francfort-sur-le-Main, 1987.

Krohn, Helga, Die Juden in Hamburg, 1800-1850, Francfort-sur-le-Main, 1967.

Lowenstein Steven M., "The Yiddish Written Word in Nineteenth-Century Germany ", in LeoBaeck-Institute Year Book, 24/1979, 178-192.

Löwenstein Leopold, Geschichte der Juden in der Kurpfalz. Nach gedruckten und ungedruckten Quellen dargestellt, Francfort-sur-le-Main, 1895.

Paucker, Arnold, "Zur Problematik einer jüdischen Abwehrstrategie in der deutschen Gesellschaft ", in Werner Mosse, Arnold Paucker (Hgg.), Juden im Wilhelminischen Deutschland, Tübingen, 1976, 479-558.

Polenz, Peter von (1991), "Deutsche Sprachgeschichte vom Spätmittelalter bis zur Gegenwart ", Band I : Einführung, Grundbegriffe, Deutsch in der frühbürgerlichen Zeit, Berlin, New York.

Polenz, Peter von (1994), Deutsche Sprachgeschichte vom Spätmittelalter bis zur Gegenwart, Bd. II : 17. und 18. Jahrhundert, Berlin, New York.

Polenz, Peter von (1999), Deutsche Sprachgeschichte vom Spätmittelalter bis zur Gegenwart, Bd. III : 19. und 20. Jahrhundert, Berlin, New York.

Richarz, Monika (Hg.), Jüdisches Leben in Deutschland. Selbstzeugnisse zur Sozialgeschichte, 1780-1871, New York, 1976.

Richarz, Monika, "Juden, Wissenschaft und Universitäten ", in Walter Grab (Hg.), Gegenseitige Einflüsse deutscher und jüdischer Kultur von der Epoche der Aufklärung bis zur Weimarer Republik (= Jahrbuch des Instituts für Deutsche Geschichte Universität, Tel Aviv, Beiheft 4), Tel Aviv, 1982, 55-73.

Rosenthal, B., "Die jüdischen Volksschulen in Baden", in Gedenkbuch zum hundertfunfundzwanzigährigen Bestehen des Oberrats der Israeliten Badens, Frankfurt am Main, 1934, 125-165.

Schorsch, Ismar, The Religious Parameters of Wissenschaft - Jewish Academics at Prussian Universities, LeoBaeck-Institute-Year Book, XXV/1980, 3-19.

Stern, Selma, Der Preußische Staat und die Juden. Dritter Teil (Die Zeit Friedrichs des Großen): 1. Abteilung : "Darstellung "; 2. Abteilung: "Akten », 1. und 2. Halbband, Tübingen, 1971.

Storost, Jürgen, "Jacob Grimm und die Schleswig-Holstein-Frage: $\mathrm{Zu}$ den Kontroversen von 1850 ", in Brüder-Grimm-Gedenken, Bd. 8, hg. von Ludwig Denecke, Marburg, 1988, 6480.

Thomann Tewarson, Heidi, "Ich bin darin der erste Ignorant der Welt! der dabei so viel auf Kenntnisse hält ", Zum Bildungsweg Rahel Levins, in Barbara Hahn, Ursula Isselstein (Hrsg.): Rahel Levin Varnhagen. = Zs. für Literaturwissenschaft und Linguistik Beiheft, 14, Göttingen, 1987, S. 141-151.

Toury, Jacob, Der Eintritt der Juden ins deutsche Bürgertum, Eine Dokumentation, Tel Aviv, 1972.

Toury, Jacob, "Die Sprache als Problem der jüdischen Einordnung im deutschen Kulturraum ", in Walter Grab (Hrsg.), Gegenseitige Einflüsse deutscher und jüdischer. Kultur von der Epoche der Aufklärung bis zur Weimarer Republik, Jahrbuch des Instituts für deutsche Geschichte der Univ., Tel Aviv, Beiheft 4, Tel Aviv, 1982, 75-96.

Wagner, Richard, Das Judenthum in der Musik, unter d. Pseudonym "Karl Freigedank ", 1850, 2. Aufl., 1869.

Wiener, Max, Jüdische Religion im Zeitalter der Emanzipation, Berlin, 1933. 